\title{
Ventilação Mecânica Não-Invasiva com Pressão Positiva
}

\author{
Noninvasive Mechanical Ventilation with Positive Pressure
}

\author{
Guilherme P. P. Schettino ${ }^{1}$, Marco Antonio Soares Reis², Filomena Galas ${ }^{3}$, Marcelo Park, \\ Suelene Aires Franca ${ }^{5}$, Valdelis Novis Okamoto ${ }^{6}$, Carlos R. R. Carvalho ${ }^{7}$
}

\section{RESUMO}

JUSTIFICATIVA E OBJETIVOS: Em 2000, foi publicado o Il Consenso Brasileiro de Ventilação Mecânica. Desde então, o conhecimento na área da ventilação mecânica avançou rapidamente, com a publicação de numerosos estudos clínicos que acrescentaram informações importantes para o manejo de pacientes críticos em ventilação artificial. Além disso, a expansão do conceito de Medicina Baseada em Evidências determinou a hierarquização das recomendações clínicas, segundo o rigor metodológico dos estudos que as embasaram. Essa abordagem explícita vem ampliando a compreensão e a aplicação das recomendações clínicas. Por esses motivos, a AMIB - Associação de Medicina Intensiva Brasileira - e a SBPT - Sociedade Brasileira de Pneumologia e Tisiologia - julgaram conveniente a atualização das recomendações descritas no Consenso anterior. Dentre os tópicos selecionados a Ventilação Mecânica Não-Invasiva foi um dos temas propostos. O objetivo deste estudo foi descrever os pontos mais importantes relacionados à ventilação mecânica na forma não-invasiva (VMNI) e sugerir as principais indicações dessa modalidade.

MÉTODO: Objetivou-se chegar a um documento suficientemente sintético, que refletisse a melhor evidência disponível na literatura. A revisão bibliográfica baseouse na busca de estudos através de palavras-chave e

1. UTI do Hospital Sírio-Libanês e UTI-Respiratória do Hospital das Clínicas da Faculdade de Medicina da Universidade de São Paulo (SP)

2. Hospital Universitário São José, Belo Horizonte (MG)

3. UTI do Hospital Sírio-Libanês e Divisão de Anestesia do Hospital das Clínicas da Faculdade de Medicina da Universidade de São Paulo (SP)

4. UTI da Disciplina de Emergências Clínicas do Hospital das Clínicas da Faculdade de Medicina da Universidade de São Paulo (SP)

5. UTI do Hospital das Clínicas da Universidade Federal de Goiás (GO)

6. UTI do Hospital AC Camargo (SP)

7. UTI-Respiratória do Hospital das Clínicas da Faculdade de Medicina da Universidade de São Paulo (SP)

(C)Associação de Medicina Intensiva Brasileira, 2007 em sua gradação conforme níveis de evidência. As palavras-chave utilizadas para a busca foram: Ventilação mecânica não invasiva: Non-invasive mechanical ventilation.

RESULTADOS: São apresentadas recomendações quanto à utilização da VMNI nas diversas formas de insuficiência respiratória e no desmame da ventilação mecânica.

CONCLUSÕES: A VMNI está indicada como o tratamento preferencial na exacerbação da DPOC, assim como, na assistência de pacientes em edema agudo de pulmão.

Unitermos: agudização da DPOC, edema agudo de pulmão, recomendação, ventilação mecânica não-invasiva

\section{SUMMARY}

BACKGROUND AND OBJECTIVES: The II Brazilian Consensus Conference on Mechanical Ventilation was published in 2000. Knowledge on the field of mechanical ventilation evolved rapidly since then, with the publication of numerous clinical studies with potential impact on the ventilatory management of critically ill patients. Moreover, the evolving concept of evidence - based medicine determined the grading of clinical recommendations according to the methodological value of the studies on which they are based. This explicit approach has broadened the understanding and adoption of clinical recommendations. For these reasons, AMIB - Associação de Medicina Intensiva Brasileira and SBPT - Sociedade Brasileira de Pneumologia e Tisiologia - decided to update the recommendations of the II Brazilian Consensus. Non-Invasive Mechanical ventilation has been one of the updated topics. Describe the most important topics on the non-invasive mechanical ventilation and suggest the main therapeutic approaches of this modality.

METHODS: Systematic review of the published literature and gradation of the studies in levels of evidence, using 
the keywords "non-invasive mechanical ventilation".

RESULTS: Recommendations on the non-invasive mechanical ventilation during respiratory failure and weaning are presented.

CONCLUSIONS: Non-invasive mechanical ventilation is the main form of ventilatory support during exacerbation of chronic obstructive pulmonary disease and in acute pulmonary edema patients.

Key Words: acute pulmonary edema, COPD, non-invasive mechanical ventilation, recommendation.

\section{INTRODUÇÃO}

O uso da ventilação mecânica não-invasiva com pressão positiva (VMNI) para o tratamento de pacientes com insuficiência respiratória aguda ou crônica agudizada foi, certamente, um dos maiores avanços da ventilação mecânica nas últimas duas décadas. Apesar do seu uso ser relativamente recente, o grande número de séries de casos, ensaios clínicos aleatórios, metanálises ou revisões sistemáticas, assim como conferências de consenso e diretrizes publicadas até o presente momento, tornaram a aplicação dessa técnica mais "baseada em evidências" do que provavelmente qualquer outra medida de suporte ventilatório ${ }^{1,2}$. Hoje não há dúvidas de que o uso da VMNI em grupos selecionados de pacientes, como, por exemplo, pacientes com exacerbação de doença pulmonar obstrutiva crônica (DPOC), é responsável pela diminuição da necessidade de intubação, mortalidade e custos do tratamento, motivo pelo qual o seu uso vem se tornando cada vez mais freqüente.

A seguir serão descritas as melhores evidências e recomendações para o uso da VMNI em ambiente hospitalar no tratamento de pacientes adultos com insuficiência respiratória aguda ou insuficiência respiratória crônica agudizada.

\section{ASPECTOS TÉCNICOS PARA USO DA VENTILAÇÃO MECÂNICA NÃO-INVASIVA INTERFACES}

As máscaras nasais ou oronasais são as interfaces mais freqüentemente utilizadas para a aplicação da VMNI no ambiente hospitalar. A máscara nasal é, provavelmente, a interface mais confortável, porém a resistência das narinas ao fluxo de ar e a presença do vazamento de ar pela boca podem limitar o seu uso em alguns pacientes ${ }^{3-5}$. A máscara oronasal, também conhecida como facial, é a interface mais utilizada para pacientes com insuficiência respiratória aguda, permitindo maior volume corrente quando comparada com a máscara nasal e, conseqüentemente, correção mais rápida das trocas gasosas ${ }^{6}$. Apesar dessas vantagens teóricas, não existe evidência suficiente para recomendar o uso da máscara oronasal ao invés da nasal para pacientes com insuficiência respiratória aguda ${ }^{7}$. Máscaras com orifício de exalação na própria máscara podem diminuir a reinalação de $\mathrm{CO}_{2}$ quando comparadas com o uso de orifícios de exalação no circuito único dos ventiladores de $\mathrm{VMNI}^{8}$.

$\mathrm{Na}$ tentativa de melhorar o conforto e a tolerância dos pacientes durante a VMNI, dispõe-se atualmente novas interfaces, como, por exemplo, a máscara facial total e o capacete. A máscara facial total tem a vantagem de diminuir o vazamento e possibilitar o uso de maiores pressões inspiratórias. Maior área de contato entre a máscara e a face do paciente pode diminuir as lesões de pele relacionadas ao uso da máscara e tornar o seu uso mais confortável ${ }^{9}$. Schettino e col. ${ }^{8}$ demonstraram que apesar do maior volume interno de ar nessa máscara, a reinalação de $\mathrm{CO}_{2}$ durante o uso da máscara facial total é semelhante à das máscaras oronasais. Os capacetes têm a vantagem de eliminar o contato da interface com a face do paciente, evitando assim, complicação mais freqüente da VMNI, que é a lesão de pele. O grande espaço-morto dos capacetes e a sua parede muito complacente levam, respectivamente, à reinalação de $\mathrm{CO}_{2}$ e à necessidade do uso de maiores valores de pressão inspiratória para garantir a correção das trocas gasosas ${ }^{10}$. O ruído interno dos capacetes pode ser um grande limitante para o seu uso ${ }^{11}$.

\section{VENTILADORES E MODOS VENTILATÓRIOS}

Teoricamente, qualquer ventilador mecânico e modo ventilatório podem ser utilizados para a ventilação não invasiva, desde que o seu funcionamento não seja prejudicado pela presença de vazamento.

Os ventiladores específicos para VMNI têm como característica principal a presença de um circuito único, por onde ocorrem tanto a inspiração como a expiração. Um orifício localizado na porção distal desse circuito é obrigatório para minimizar a reinalacão de $\mathrm{CO}_{2}$ durante a inspiração ${ }^{8,12,13}$. Esse orifício faz com que haja um vazamento contínuo de ar pelo circuito, eliminando $\mathrm{O} \mathrm{CO}_{2}$ exalado pelo paciente durante a expiração. Por esse motivo, os ventiladores específicos para VMNI foram desenhados para funcionar na presença de vazamento. Tolerância ao vazamento, boa 
sincronia paciente-ventilador e preço competitivo são as principais vantagens desses ventiladores, quando comparados aos ventiladores de UTI ${ }^{14-16}$. Restrição de alguns modos ventilatórios, limitação de alarmes e dificuldade para o ajuste da $\mathrm{FIO}_{2}$ são algumas das limitações desses aparelhos específicos para VMNI, porém alguns modelos já apresentam esses requisitos. Os novos ventiladores de UTI estão sendo adaptados para funcionarem tanto durante a ventilação invasiva quanto não invasiva. Algoritmos para a compensação automática de vazamento e a possibilidade do ajuste do critério de ciclagem da fase inspiratória para a expiração durante a pressão de suporte são exemplos de mudanças para facilitar o uso dos ventiladores de UTI para o suporte ventilatório não-invasivo. Entretanto, não existe, até o momento, nenhum estudo comparando o uso de ventiladores de UTI com aqueles específicos para VMNI para o suporte ventilatório não-invasivo em pacientes com insuficiência respiratória aguda.

Existem relatos do uso bem sucedido de VMNI com emprego dos modos CPAP, volume controlado, pressão controlada, pressão de suporte e ventilação assistida proporcional (PAV).

Para pacientes com DPOC agudizada, pressão de suporte e PEEP (PS + PEEP ou inspiratory positive airway pressure, também conhecido como IPAP, nível acima da EPAP - expiratory positive airway pressure) é o modo ventilatório utilizado na maioria dos estudos publicados até o momento, sendo por isto o modo ventilatório recomendado pela maioria dos autores ${ }^{17}$. Nessa modalidade, a PS (ou IPAP) deve ser ajustada para gerar um volume-corrente por volta de 6 a $8 \mathrm{~mL} /$ $\mathrm{kg}$ e freqüência respiratória $<30 / \mathrm{min}$. Valor da PEEP (ou EPAP) deve ser inferior ao da PEEP intrínseca (devido à dificuldade de medir a PEEP intrínseca durante a ventilação espontânea, sugere-se o uso da PEEP/ EPAP inicialmente em torno de $6 \mathrm{cmH}_{2} \mathrm{O}$ ). A ventilação assistida proporcional (PAV) pode ser uma alternativa a PS + PEEP para VMNI na DPOC agudizada, como demonstrado em alguns estudos clínicos ${ }^{18,20}$.

CPAP (continuous positive airway pressure) é um modo ventilatório empregado com freqüência para o suporte ventilatório não invasivo de pacientes com edema agudo de pulmão (EAP). Nessa situação, acredita-se que se deva utilizar um valor mínimo de $10 \mathrm{cmH}_{2} \mathrm{O}$ para garantir os benefícios hemodinâmicos e ventilatórios da CPAP. Deve-se lembrar que a CPAP não é capaz de aumentar a ventilação alveolar, motivo pelo qual, na presença de hipercapnia, é dada pre- ferência ao uso da ventilação não-invasiva com dois níveis de pressão ${ }^{21,22}$. Fu e col. ${ }^{23}$ demonstraram que a CPAP obtida com gerador de fluxo tem funcionamento semelhante à CPAP de ventiladores mecânicos para aplicação não-invasiva.

\section{LIMITAÇÕES PARA O USO DA VENTILAÇÃO MECÂ- NICA NÃO-INVASIVA}

A adequada seleção dos pacientes ao uso da ventilação não-invasiva é o primeiro passo para o sucesso da técnica (Quadro 1). Como a VMNI é uma modalidade de suporte ventilatório parcial e sujeita a interrupções, essa técnica não deve ser utilizada em pacientes totalmente dependentes da ventilação mecânica para se manterem vivos. A cooperação do paciente é importante para o sucesso da VMNI, tornando o seu uso limitado nos pacientes com rebaixamento do estado de consciência ou com agitação. Da mesma forma, pela inexistência de uma prótese traqueal a VMNI só deve ser utilizada naqueles pacientes capazes de manter a permeabilidade da via aérea superior, assim como a integridade dos mecanismos de deglutição e a capacidade de mobilizar secreções. Instabilidade hemodinâmica grave, caracterizada pelo uso de aminas vasopressoras, e disritmias complexas são consideradas contra-indicações para o uso da VMNI pela maioria dos autores. Pacientes com distensão abdominal ou vômitos não devem utilizar VMNI pelo risco de aspiração. Pósoperatório imediato de cirurgia do esôfago é contraindicação para VMNI, entretanto dúvidas persistem acerca da segurança do seu uso no pós-operatório de cirurgias gástricas. Trauma de face, lesão aguda e/ou sangramento de via aérea são também consideradas limitações para o uso da VMNI.

Quadro 1 - Contra-Indicações para o Uso da Ventilação Mecânica Não-Invasiva com Pressão Positiva

Diminuição da consciência, sonolência, agitação, confusão ou
recusa do paciente
Instabilidade hemodinâmica com necessidade de medicamen-
to vasopressor, choque (pressão arterial sistólica < $90 \mathrm{mmHg}$ ),
disritmias complexas
Obstrução de via aérea superior ou trauma de face
Tosse ineficaz ou incapacidade de deglutição
Distensão abdominal, náuseas ou vômitos
Sangramento digestivo alto
Infarto agudo do miocárdio
Pós-operatório recente de cirurgia de face, via aérea superior
ou esôfago
Uso de VMNI é controverso: pós-operatório de cirurgia gástri-
ca, gravidez


INDICAÇÕES PARA O USO DA VENTILAÇÃO MECÂNICA NÃO-INVASIVA

A seguir serão descritas as recomendações e os graus de evidência para o emprego da VMNI nas condições mais freqüentes para o seu uso em ambiente hospitalar, com enfoque na redução da taxa de intubação e da mortalidade. Caso existam estudos com diferentes níveis de evidência e concordantes em recomendar ou não o uso da VMNI, serão descritos somente os estudos com os maiores níveis de evidências.

\section{VENTILAÇÃO MECÂNICA NÃO-INVASIVA NA EXA- CERBAÇÃO DA DPOC}

Recomendação: VMNI deve ser utilizada como tratamento de primeira escolha para pacientes com agudização da DPOC, especialmente para aqueles pacientes com exacerbação grave, caracterizada pela presença de acidose respiratória $(\mathrm{pH}<7,35)$ que persiste a despeito de tratamento médico máximo associado a oxigenoterapia controlada. O uso de VMNI diminui a necessidade de intubação e reduz a mortalidade hospitalar desses pacientes. Por esses motivos, essa intervenção deve estar disponível nos hospitais que atendam pacientes com exacerbação de DPOC.

\section{Grau de Recomendação: A}

Comentário: A evidência mais forte para o benefício do uso da VMNI, tanto para a diminuição da necessidade de intubação quanto para a redução da mortalidade, é no tratamento da exacerbação da DPOC. Existem, até o momento, três metanálises ${ }^{24,26}$ baseadas em estudos controlados e aleatórios, realizados exclusivamente em pacientes com DPOC agudizada ${ }^{27-34}$ que comprovam o benefício do uso da VMNI para diminuir tanto a necessidade de intubação quanto a mortalidade hospitalar, quando a ventilação não invasiva é comparada com o tratamento convencional para esses pacientes. Entretanto, a análise de Keenan e col. ${ }^{25}$ reportou que esses benefícios não foram demonstrados em pacientes com exacerbações mais leves de DPOC, enfatizando o conceito de que a VMNI é indicada para pacientes com exacerbações mais graves, acompanhadas de hipercapnia e acidose respiratória. Esse conceito foi recentemente questionado por estudo aleatório chinês, com 342 pacientes, em que a VMNI foi aplicada a pacientes em fase precoce (entre 24 e 48h) de exacerbação aguda de DPOC que demonstrou benefício também para os casos com menor alteração de $\mathrm{pH}$ e $\mathrm{PaCO}_{2}$. Nesse estudo, houve menor taxa de intubação no grupo que recebeu $\mathrm{VMNI}(4,7 \%$ versus $15,2 \%$ no grupo controle, $\mathrm{p}=0,002)$. A diferença de mortalidade entre os grupos, porém, não foi significativa $(4,1 \% \text { versus } 7 \%)^{35}$.

A tabela 1, adaptada de Hess ${ }^{17}$, descreve o benefício da VMNI em diminuir a necessidade de intubação e mortalidade para pacientes com exacerbação da DPOC segundo as referidas metanálises.

\section{VMNI NA EXACERBAÇÃO DA ASMA}

Recomendação: VMNI pode ser utilizada em conjunto com o tratamento medicamentoso convencional para o cuidado de pacientes selecionados com exacerbação aguda e grave da asma.

\section{Grau de Recomendação: B}

Comentário: O sucesso do emprego da VMNI no tratamento da agudização da DPOC fez com que o seu uso fosse também tentado no tratamento da exacerbação da asma. Meduri e col. ${ }^{36}$ descreveram o uso da VMNI em 17 pacientes com exacerbação aguda de asma e relataram que a intubação foi necessária em apenas três desses casos. Soroksky e col. ${ }^{37}$ realizaram

Tabela 1 - Ventilação Mecânica Não-Invasiva para Exacerbação da DPOC: Metanálises

\begin{tabular}{|c|c|c|c|}
\hline Autores & Estudos (n) & Intubação & Mortalidade \\
\hline Lightowler e col. ${ }^{24}$ & 8 & $\begin{array}{c}\text { Risco relativo }=0,42 \\
\text { IC } 95 \%=0,31 \text { a } 0,59\end{array}$ & $\begin{array}{l}\text { Risco relativo }=0,41 \\
\text { IC } 95 \%=0,26 \text { a } 0,64\end{array}$ \\
\hline Keenan e col. ${ }^{25}$ & 15 & $\begin{array}{c}\text { Exacerbação grave*: } \\
\text { Redução do risco = } 34 \% \\
\text { IC } 95 \%=22 \% \text { a } 46 \% \\
\text { Exacerbação não grave: } \\
\text { Redução do risco = 0\% } \\
\text { IC } 95 \%=-11 \% \text { a } 11 \%\end{array}$ & $\begin{array}{c}>\text { Exacerbação grave* } \\
\text { Redução do risco = } 12 \% \\
\text { IC } 95 \%=6 \% \text { a } 18 \% \\
\text { Exacerbação não grave: } \\
\text { Redução do risco }=2 \% \\
\text { IC } 95 \%=-8 \% \text { a } 12 \%\end{array}$ \\
\hline Ram e col. ${ }^{26}$ & 14 & $\begin{array}{l}\text { Risco relativo }=0,41 \\
\text { IC } 95 \%=0,33 \text { a } 0,53\end{array}$ & $\begin{array}{l}\text { Risco relativo }=0,52 \\
\text { IC } 95 \%=0,35 \text { a } 0,76\end{array}$ \\
\hline
\end{tabular}

Legenda: $\mathrm{n}$ : número de estudos incluídos na metanálise; IC: intervalo de confiança; * Exacerbação grave: definida como acidose respiratória (pH < 7,30) ou mortalidade hospitalar observada no grupo controle $>10 \%$. Observação: Risco relativo $<1$ indica proteção. 
um estudo aleatório em 30 pacientes com agudização de asma, que incluía o uso de uma manobra de "VMNIplacebo" no grupo controle (que consistiu na utilização de VMNI com pressões ins e expiratória de $1 \mathrm{cmH}_{2} \mathrm{O}$ ), para avaliar o benefício da VMNI nessa condição. Esses autores demonstraram que os pacientes que receberam VMNI por três horas consecutivas apresentaram melhora mais rápida dos sintomas, aumento do $\mathrm{VEF}_{1}$ e diminuição da necessidade de internação em relação ao grupo controle. Revisão sistemática recente da Cochrane $^{38}$ sobre o tema não encontrou nenhum outro estudo relevante, a não ser o já descrito, concluindo que o uso da VMNI para o tratamento da exacerbação grave da asma parece promissor, porém o único estudo publicado, devido ao limitado número de pacientes avaliados, não permite a recomendação definitiva para o seu uso.

\section{VENTILAÇÃO MECÂNICA NÃO-INVASIVA NO EDE- MA PULMONAR CARDIOGÊNICO}

\section{CPAP NO EDEMA PULMONAR CARDIOGÊNICO}

Recomendação: O uso de CPAP é seguro e diminui a necessidade de intubação para pacientes com edema agudo de pulmão, devendo ser aplicado precocemente e em conjunto com a terapia medicamentosa convencional.

\section{Grau de Recomendação: A}

Comentário: As evidências para a utilização de CPAP em insuficiência respiratória aguda vêm de três ensaios clínicos aleatório ${ }^{39-41}$ que compararam CPAP com tratamento convencional em pacientes com edema pulmonar cardiogênico (EPC). Rasanen e col. ${ }^{39}$ compararam o tratamento convencional isolado ou associado ao uso de pressão positiva contínua em vias aéreas (CPAP $=10 \mathrm{cmH}_{2} \mathrm{O}$ ), para pacientes com edema pulmonar de origem cardíaca. Nesse estudo, houve apenas melhora mais rápida da hipoxemia no grupo que usou CPAP. No estudo de Bersten ${ }^{40}, 39$ pacientes com EPC e alto risco de intubação, caracterizado pela presença de hipercapnia, foram aleatorizados para receber tratamento convencional, associada ou não a CPAP $=10 \mathrm{cmH}_{2} \mathrm{O}$. Além da melhora rápida da hipercapnia e da hipoxemia, nenhum paciente que recebeu CPAP foi intubado, versus $35 \%$ daqueles em tratamento convencional. $\mathrm{Na}$ casuística de Lin e col. ${ }^{41}$, com 100 pacientes, foi aplicado CPAP em níveis progressivamente maiores, variando de 2,5 até $12,5 \mathrm{cmH}_{2} \mathrm{O}$, num intervalo de duas horas e meia. $O$ resultado foi concordante com o estudo de Bersten ${ }^{40}$. A análise conjunta desses três es- tudos $^{42}$ mostrou redução na necessidade de intubação (diferença de risco $=-26 \%$, IC 95\% $=-13 \%$ a $-38 \%$ ) e tendência à redução na mortalidade (diferença de risco $=-6,6 \%$, IC $95 \%=3 \%$ a $-16 \%$ ) com o uso do CPAP. Em estudo mais recente, L'Her e col. ${ }^{43}$ demonstraram, em 89 pacientes consecutivos, com idade acima de 80 anos, a superioridade do uso de CPAP $=10 \mathrm{cmH}_{2} \mathrm{O} \mathrm{em}$ termos de redução de complicações (17 pacientes no grupo controle versus 4 pacientes no grupo CPAP; $p=$ 0,002 ) e de mortalidade com 48 horas de inclusão (tratamento convencional $=24 \%$ versus $\mathrm{CPAP}=7 \% ; \mathrm{p}=$ $0,017)$. Porém, não houve diferença nas taxas de mortalidade hospitalar entre os dois grupos de estudo.

\section{VMNI COM DOIS NÍVEIS DE PRESSÃO NO EDEMA PULMONAR CARDIOGÊNICO}

Recomendação: Pacientes com hipercapnia associada à hipoxemia parecem ser os que mais se beneficiam do uso da VMNI com PEEP acrescido de pressão de suporte (PEEP + PS) no tratamento do EPC. $\mathrm{O}$ uso de pressão expiratória de $10 \mathrm{cmH}_{2} \mathrm{O}$ parece ser o ponto chave do benefício respiratório/hemodinâmico para pacientes com edema agudo dos pulmões de origem cardíaca, tanto durante o uso do CPAP, quanto na VMNI com PEEP + PS.

\section{Grau de Recomendação: B \\ Comentários:}

a) VMNI COM PEEP E PS versus CPAP: Não é claro, no momento, se a VMNI com PEEP e PS é superior a CPAP no tratamento do EPC ${ }^{44,45}$. No primeiro estudo utilizando VMNI com PEEP e PS para o tratamento do EPC, Mehta e col. ${ }^{46}$ compararam 14 pacientes em uso de VMNI com PS $=15 \mathrm{cmH}_{2} \mathrm{O}+$ PEEP $=5 \mathrm{cmH}_{2} \mathrm{O}$, com 13 pacientes em uso de CPAP $=10 \mathrm{cmH}_{2} \mathrm{O}$. Houve, na análise intragrupo, diminuição mais rápida na pressão arterial, freqüências cardíaca e respiratória no grupo aleatório para o uso de VMNI com PEEP e PS. A taxa de intubação foi semelhante, porém o resultado que tornou esse estudo polêmico foi a elevada taxa de infarto agudo do miocárdio no grupo VMNI com PEEP e PS (71\% versus $31 \%)$, o que resultou na interrupção do estudo. No ensaio clínico de Bellone e col. ${ }^{47}$, 36 pacientes com edema agudo dos pulmões e hipercapnia $\left(\mathrm{PaCO}_{2}>45 \mathrm{mmHg}\right)$ foram aleatorizados para tratamento com CPAP $=10 \mathrm{cmH}_{2} \mathrm{O}$ ou VMNI com PEEP = $5 \mathrm{cmH}_{2} \mathrm{O}$ e PS $=15 \mathrm{cmH}_{2} \mathrm{O}$ ). Nesse estudo, não houve diferença entre os grupos com relação a tempo para melhora clínica, taxa de intubação e mortalidade hospitalar. Níveis de troponina I sérica e de infarto agudo do miocárdio foram semelhantes nos dois grupos. 
b) VMNI COM PEEP E PS versus OXIGENOTERA-

PIA: Também não é claro, no momento, se VMNI com PEEP e PS é superior ao tratamento convencional do EPC (o benefício parece existir em pacientes com hipercapnia). No estudo aleatório de Masip e col. ${ }^{21}$, comparando o tratamento convencional, associado ou não ao uso da VMNI com PEEP e PS, houve meIhora da hipoxemia, redução da taxa de intubação (5\% versus $33 \%)$ e recuperação mais rápida da hipercapnia no grupo aleatório para VMNI com PEEP e PS. Os efeitos benéficos do uso da VMNI com PEEP e PS foram mais evidentes para aqueles pacientes hipercápnicos na aleatorização. A incidência de infarto agudo do miocárdio foi semelhante em ambos os grupos. Nava e col. ${ }^{48}$ estudaram 130 pacientes que receberam VMNI com PEEP $=5 \mathrm{cmH}_{2} \mathrm{O}$ e PS = $15 \mathrm{cmH}_{2} \mathrm{O}$ ou tratamento convencional. Apenas os pacientes com hipercapnia $\left(\mathrm{PaCO}_{2}>45 \mathrm{mmHg}\right)$ alocados para uso da VMNI com PEEP e PS tiveram redução na taxa de intubação $(6 \%$ versus $28 \%, p=$ $0,015)$. A taxa de infarto agudo do miocárdio foi igual nos dois grupos.

c) VMNI COM PEEP E PS versus CPAP versus OXIGENOTERAPIA: No estudo de Crane ${ }^{49}, 60$ pacientes foram aleatorizados para tratamento convencional, CPAP $=10 \mathrm{cmH}_{2} \mathrm{O}$ ou VMNI com PEEP $=5 \mathrm{cmH}_{2} \mathrm{O}$ e PS $=15 \mathrm{cmH}_{2} \mathrm{O}$. Houve melhora semelhante entre os grupos na oxigenação, acidose e diminuição da freqüência respiratória. As taxas de intubação e de infarto agudo do miocárdio também foram semelhantes entre os grupos. No entanto, a mortalidade hospitalar maior nos grupos controle e VMNI com PEEP e PS, comparados com o grupo CPAP $(30 \%$ no grupo controle, $15 \%$ no grupo VMNI com PEEP e PS e $0 \%$ no grupo (PAP). No estudo de Park e col. ${ }^{22}, 80$ pacientes com EPC foram aleatorizados para receber tratamento convencional (oxigenoterapia com $\mathrm{FIO}_{2}=$ $0,5), \mathrm{CPAP}=10 \mathrm{cmH}_{2} \mathrm{O}$ ou VMNI com PS $=15 \mathrm{cmH}_{2} \mathrm{O}$ $+\mathrm{PEEP}=10 \mathrm{cmH}_{2} \mathrm{O}$. Além da melhora equivalente dos sinais vitais nos grupos CPAP e VMNI com PEEP + PS, observaram melhora nitidamente superior em relação ao grupo tratado de forma convencional, a taxa de intubação foi reduzida de $42 \%$ durante o tratamento convencional para $7 \%$ em ambos os grupos que usaram pressão positiva não-invasiva, havendo também redução da mortalidade em 14 dias a favor dos pacientes que utilizaram pressão positiva não-invasiva (CPAP e VMNI com PEEP + PS). Não houve aumento da incidência de infarto agudo do miocárdio nessa população.

\section{VENTILAÇÃO MECÂNICA NÃO-INVASIVA NA INSU- FICIÊNCIA RESPIRATÓRIA HIPOXÊMICA}

Recomendação: A VMNI pode ser benéfica na insuficiência respiratória hipoxêmica, porém seu uso deve ser cauteloso.

\section{Grau de Recomendação: B}

Comentário: A insuficiência respiratória hipoxêmica pode ser causada por várias doenças, com características fisiopatológicas e curso clínico distintos, o que torna a avaliação dos benefícios do uso da VMNI para o tratamento de pacientes nessa condição complexa e controversa ${ }^{1,17,50-53 .}$ Alguns estudos, apesar de considerarem casuísticas heterogêneas, mostram benefícios do uso da VMNI em reduzir a necessidade de intubação, complicações associadas à ventilação mecânica e mesmo mortalidade quando a VMNI é comparada com tratamento convencional, ou mesmo com a ventilação invasiva, para o cuidado de pacientes com insuficiência respiratória hipoxêmica. Em um dos primeiros ensaios clínicos de VMNI na insuficiência respiratória hipoxêmica, Antonelli e col. 54, em 1998, compararam 64 pacientes com insuficiência respiratória aguda de diversas etiologias que foram submetidos à ventilação mecânica invasiva ou VMNI. O seu uso foi tão efetivo quanto a ventilação convencional para a correção da hipoxemia, mas o grupo VMNI apresentou menor tempo de ventilação mecânica e alta mais precoce da UTI, além de apresentar menos complicações associadas à ventilação mecânica. Ferrer e col..$^{55}$ publicaram estudo realizado em três centros na Espanha, no qual se comparou o uso da VMNI com o tratamento convencional baseado no uso de máscara Venturi com altas $\mathrm{FIO}_{2}$ em 105 pacientes com insuficiência respiratória aguda grave de diversas etiologias. Observaram que a VMNI se associou à menor necessidade de intubação (13,25\% versus $28,52 \%)$, menor incidência de choque séptico $(6,12 \%$ versus $17,31 \%)$, menor mortalidade na UTI ( $9,18 \%$ versus $21,39 \%)$, além de maior sobrevida em 90 dias.

Existem três revisões sistemáticas ou metanálises sobre o uso da VMNI na insuficiência respiratória hipoxêmica ${ }^{56-58}$ (Tabela 2). Na mais recente delas, Keenan e col. ${ }^{58}$ avaliaram os estudos aleatórios publicados até outubro de 2003 sobre o uso da VMNI em pacientes com insuficiência respiratória aguda hipoxêmica não relacionada ao edema agudo de pulmão. Os resultados dessa revisão sistemática comprovam que o uso da VMNI está associado à redução na necessidade de intubação (redução de risco = 24\% [IC 95\%: 8\% a 36\%]), redução nos dias de permanência na UTI em 1,9 dia (IC 
95\%: 1 a 2,9 dias), redução da mortalidade na UTI 16\% (IC 95\%: 5\% a 27\%) e a uma tendência à redução da mortalidade hospitalar de 10\% (IC 95\%: -7\% a - 27\%). Os autores dessa revisão sistemática salientam, entretanto, que os resultados apresentados sejam avaliados com cuidado, pois é possível que, dentro da população heterogênea de pacientes com insuficiência respiratória aguda, existam subgrupos que se beneficiem com a VMNI, como, por exemplo, pacientes imunossuprimidos ou pós-operatório de toracotomia e outros, nos quais o seu efeito benéfico ainda não foi comprovado. Aconselham ainda que novos estudos aleatórios sejam realizados em grupos específicos de pacientes com insuficiência respiratória aguda hipoxêmica.

Tabela 2 - Ventilação Mecânica Não-Invasiva para Insuficiência Respiratória aguda Hipoxêmica: Metanálises

\begin{tabular}{lccc}
\hline Autores Estudos & Intubação & Mortalidade \\
& $(\mathrm{n})$ & & \\
\hline Keenan & 7 & Odds ratio $=0,20$ & Odds ratio $=0,29$ \\
e col. ${ }^{56}$ & & IC 95\% $=0,11$ a 0,36 & IC 95\% $=0,15$ a 0,59 \\
Peter e & 15 & $\begin{array}{c}\text { Diferença } \\
\text { de risco }=-0,19\end{array}$ & $\begin{array}{c}\text { Diferença de risco }= \\
\text { col. }^{57}\end{array}$ \\
& & IC 95\% $=-0,28$ a $-0,09$ & IC 95\% $=-0,16$ a $-0,01$ \\
Keenan & 14 & Risco relativo $=0,41$ & Risco relativo $=0,52$ \\
e col. ${ }^{58}$ & & IC 95\% $=0,33$ a 0,53 & IC 95\% $=0,35$ a 0,76 \\
\hline
\end{tabular}

$\mathrm{N}$ = número de estudos incluídos na metanálise; IC: intervalo de confiança.

\section{VMNI NA INSUFICIÊNCIA RESPIRATÓRIA HIPOXÊ- MICA EM CONDIÇÕES ESPECÍFICAS}

Recomendação: O uso da VMNI parece ser útil para diminuir a mortalidade em subgrupos específicos de pacientes com insuficiência respiratória hipoxêmica, como, por exemplo, pacientes imunossuprimidos.

\section{Grau de Recomendação: B}

\section{Comentários:}

a) Imunossupressão e pós-transplante: A morbimortalidade da pneumonia associada à ventilação mecânica invasiva é alta para pacientes imunossuprimidos e transplantados de medula óssea ou órgãos sólidos. Antonelli e col. ${ }^{59}$, em 2000, publicaram estudo no qual avaliaram, de forma aleatorizada e controlada, a aplicação da VMNI, comparando-a com o tratamento padrão, incluindo suplementação de oxigênio administrado por máscara facial, em 40 receptores de órgãos sólidos, que desenvolveram insuficiência respiratória aguda hipoxêmica. Observaram melhora precoce e mantida da oxigenação e menor necessidade de intubação no grupo VMNI comparado com o tratamento padrão (20\% versus $70 \%)$. Também houve menor mortalidade no grupo que recebeu VMNI (20\% versus
$50 \%)$. Hilbert e col. ${ }^{60}$ compararam a aplicação precoce e intermitente de VMNI com o tratamento convencional, incluindo a suplementação de oxigênio, em 52 pacientes imunossuprimidos, a maioria deles transplantados de medula óssea, com insuficiência respiratória aguda, febre e infiltrados pulmonares. No grupo submetido à VMNI houve menor necessidade de intubação (12\% versus $20 \%$ ), menor número de complicações graves (13\% versus $21 \%$ ), o que levou à menor mortalidade na UTI (10\% versus $18 \%$ ) e hospitalar (13\% versus $21 \%)$. Também na insuficiência respiratória aguda relacionada à pneumonia por Pneumocystis carinii, em pacientes com AIDS, parece haver benefício do uso da VMNI. Confalonieri e col. ${ }^{61}$, em estudo prospectivo, tipo casocontrole, publicado em 2002, no qual 24 pacientes tratados com VMNI foram comparados com 24 pacientes tratados com ventilação mecânica invasiva demonstraram que o uso de VMNI evitou a intubação em $67 \%$ dos pacientes tratados, o que se associou com melhor sobrevida na UTI (100\% versus $38 \%$; $p=0,003$ ), no hospital e aos 60 dias da inclusão.

b) Pneumonia: Confalonieri e col. ${ }^{62}$ estudaram a aplicação de VMNI comparada ao tratamento padrão, de forma aleatória e controlada, em 56 pacientes com pneumonia comunitária grave e relacionaram a utilização de VMNI com menor necessidade de intubação ( $21 \%$ versus $50 \%, p=0,03$ ) e menor tempo de permanência na UTI (1,8 dia versus 6 dias, $p=0,04)$. Os dois grupos tiveram a mesma mortalidade hospitalar, entretanto, entre os pacientes com DPOC, aqueles aleatorizados para $\mathrm{VMNI}$ apresentaram maior sobrevida aos dois meses $(88,9 \%$ versus $37,5 \%, p=0,005)$.

c) Pós-ressecção pulmonar: Auriant e col. ${ }^{63}$ avaliaram 47 pacientes que desenvolveram IRA após cirurgia de ressecção pulmonar, aleatorizados para VMNI ou tratamento convencional, relatando menor necessidade de intubação traqueal (21\% versus $50 \%$ ) e menor taxa de mortalidade $(12,5 \%$ versus $37,5 \%)$ nos pacientes submetidos à VMNI.

d) Lesão pulmonar aguda/síndrome do desconforto respiratório agudo (LPA/SDRA): Rocker e col. ${ }^{64}$ estudaram 12 episódios de LPA/SDRA, nos quais foi empregada a VMNI, por máscara facial, utilizando dois níveis pressóricos. A taxa de sucesso foi de $50 \%$ e a sobrevida, de $70 \%$. No estudo de Ferrer e col. $^{55}$, no qual se comparou a aplicação de VMNI com altas $\mathrm{FIO}_{2}$ administradas por máscara Venturi, em pacientes com insuficiência respiratória aguda grave, havia 15 pacientes (14,5\% do total de 105 pacientes) com LPA/SDRA. Desses, sete foram aleatorizados para VMNI e oito tra- 
tados no grupo controle. Apesar dos resultados gerais do estudo terem sido favoráveis à utilização da VMNI, os desfechos nos pacientes com LPA/SDRA foram muito ruins. A taxa de intubação foi alta nos pacientes com LPA/SDRA em ambos os grupos (86\% no grupo VMNI versus $100 \%$ no grupo $\mathrm{O}_{2}$ ), assim como a mortalidade $(71 \%$ versus $87,5 \%$, respectivamente).

\section{CUIDADOS ESPECIAIS DURANTE A VMNI NA IN- SUFICIÊNCIA RESPIRATÓRIA HIPOXÊMICA}

Recomendação: Devido ao alto risco de falência da VMNI e conseqüente necessidade de intubação, pacientes com insuficiência respiratória hipoxêmica devem receber VMNI onde existam facilidades para vigilância, monitoração, intubação traqueal e ventilação invasiva.

\section{Grau de Recomendação: D}

Comentário: Entende-se que a VMNI pode ser tentada em pacientes com insuficiência respiratória hipoxêmica, desde que o paciente não apresente contra-indicações para o seu uso. No entanto, defende-se que seu uso deva ser feito em ambiente com supervisão médica constante, com possibilidade de monitoração dos sinais vitais, incluindo oximetria de pulso e com facilidades para intubação. Pacientes que necessitem de $\mathrm{FIO}_{2}>0,6$, aqueles que apresentem diminuição rápida da oxigenação $\left(\mathrm{SaO}_{2}<90 \%\right)$ após a retirada da máscara e pacientes que não apresentem melhora do quadro nas primeiras duas horas de uso da VMNI devem ser considerados para intubação eletiva e segura.

\section{VMNI EM PACIENTES TERMINAIS}

Recomendação: VMNI pode ser usada em pacientes terminais quando a causa da insuficiência respiratória for potencialmente reversível, particularmente naqueles pacientes com DPOC agudizada ou com edema pulmonar de origem cardíaca.

\section{Grau de Recomendação: B}

Comentário: O uso da VMNI pode ser útil para tratar a insuficiência respiratória de pacientes terminais, desde que a causa da insuficiência respiratória seja potencialmente reversível e não represente somente a evolução final de doença pulmonar ou extrapulmonar. Vale salientar que alguns pacientes com doenças terminais referem o desejo de não serem intubados, mas aceitam o uso da ventilação não-invasiva como forma de suporte ventilatório para o tratamento da insuficiência respiratória ou mesmo como medida paliativa para alívio da dispnéia. As evidências para o uso da VMNI em pacientes terminais são escassas e baseiam-se em estudos observacionais. Meduri e col. ${ }^{65}$ descreveram sobrevida hospitalar em $64 \%$ de 11 pacientes com DPOC agudizada, que haviam previamente recusado intubação, e que receberam VMNI para tratamento da exacerbação da doença pulmonar. Benhamou e col. ${ }^{66}$ publicaram resultados semelhantes quando a VMNI foi utilizada em 30 pacientes, a maioria deles com insuficiência respiratória hipercápnica, que haviam recusado intubação, ou que esse procedimento foi considerado inapropriado, seja pela idade ou presença de comorbidades, com taxa de sucesso de $60 \%$. O benefício do uso da VMNI para pacientes com DPOC avançado foi também sugerido por Chu e col. ${ }^{67} \mathrm{em}$ estudo com controle histórico. Em estudo observacional recente, Levy e col. ${ }^{68}$ descreveram que 1211 pacientes consecutivos que usaram VMNI para tratamento de insuficiência respiratória aguda em quatro centros americanos, 114 haviam manifestado o desejo de não serem intubados. A sobrevida hospitalar destes pacientes foi de $57 \%$, sendo a presença de hipercapnia e diagnóstico de insuficiência cardíaca associada à melhor evolução, enquanto que a presença de pneumonia ou de câncer determinava pior prognóstico. Schettino e col. ${ }^{69}$ desmostraram que o uso de VMNI em pacientes que não seriam intubados pode ser benéfico para aqueles pacientes com o diagnóstico de DPOC agudizado e insuficiência cardíaca congestiva, mas não em pacientes com insuficiência respiratória hipoxêmica, insuficiência respiratória pós-extubação ou pacientes com doença oncológica avançada. Um escore SAPS II > 35 pontos e albumina $<2,5 \mathrm{~g} / \mathrm{dL}$ foram as variáveis independentes associadas à mortalidade hospitalar desses pacientes.

\section{VMNI NO PERIODO PÓS-OPERATÓRIO IMEDIATO}

Recomendação: A VMNI pode ser utilizada com cautela para o tratamento da insuficiência respiratória hipoxêmica no período pós-operatório imediato de cirurgias abdominais e torácicas eletivas. A utilização da VMNI no período pós-operatório deve respeitar as limitações e contra-indicações para o seu uso.

\section{Grau de Recomendação: B}

Comentário: O uso da VMNI para tratamento de insuficiência respiratória hipoxêmica no período pós-operatório imediato de cirurgia abdominal e torácica eletivas está associado à diminuição da necessidade de intubação e possivelmente diminuição da mortalidade. A VMNI administrada através de máscara ou peças bucais, de forma contínua ou intermitente, tem sido utilizada isoladamente ou associada a manobras fisioterápicas para a prevenção de atelectasia e hipoxemia 
no período pós-operatório de cirurgias abdominais, porém com resultados conflitantes ${ }^{70-74}$. Existem descrições também do uso de VMNI no pós-operatório de cirurgia bariátrica, seja na tentativa de evitar atelectasia ou para tratamento de hipoventilação associada à apnéia obstrutiva do sono, tão freqüente nesses pacientes $^{75}$. Joris e col. ${ }^{76}$ demonstraram, em estudo aleatório, que 0 uso de $\mathrm{PS}=12 \mathrm{cmH}_{2} \mathrm{O}+\mathrm{PEEP}=4 \mathrm{cmH}_{2} \mathrm{O}$ nas primeiras 24 horas de pós-operatório de gastroplastia melhorou a função respiratória de pacientes obesos quando comparado com o tratamento convencional ou $\mathrm{PS}=8 \mathrm{cmH}_{2} \mathrm{O}+\mathrm{PEEP}=4 \mathrm{cmH}_{2} \mathrm{O}$. O estudo aleatório e multicêntrico de Squadrone e col. demonstrou pela primeira vez que VMNI (CPAP de 7,5 $\mathrm{cmH}_{2} \mathrm{O}$ administrado através de capacete), quando utilizada de forma precoce para o tratamento de hipoxemia $\left(\mathrm{PaO}_{2} / \mathrm{FIO}_{2}\right.$ $<300 \mathrm{mmHg}$ ) no pós-operatório imediato de cirurgia abdominal eletiva e de grande porte, incluindo gastrectomias, leva à redução da necessidade de intubação traqueal e de pneumonia nosocomial, à diminuição do tempo de internação na UTI, sem resultar em aumento de complicações associadas à cirurgia ${ }^{77}$.

O pacientes no pós-operatório de cirurgia torácica também podem se beneficiar do uso de VMNI. Aguilo e col. demonstraram, em estudo aleatório, que o uso de PS + PEEP por máscara nasal foi útil para corrigir as trocas gasosas quando comparado com tratamento convencional em pacientes no pós-operatório de toracotomia para ressecção pulmonar, sem, contudo, levar a complicações pós-operatórias como, por exemplo, fístula pleural ${ }^{78}$. Auriant e col., também em estudo aleatório, demonstraram que o uso de VMNI para o tratamento de insuficiência respiratória hipoxêmica no pós-operatório de ressecção pulmonar, está associado à menor necessidade de intubação $(20,8$ versus $50 \%)$ e mortalidade (12,5\% versus $37,5 \%$ ), quando comparado com o tratamento convencional ${ }^{63}$. Vários autores demonstraram que o uso de CPAP, ou preferencialmente PS + PEEP, é seguro e pode ser útil para melhorar a função pulmonar e as trocas gasosas no pós-operatório de cirurgia de revascularização do miocárdio, entretanto a importância clínicas desses achados necessita de confirmação $0^{79-81}$.

É importante ressaltar que, embora algumas das recomendações ou consensos publicadas até o momento incluam cirurgia do trato digestivo alto como contraindicação para VMNI, alguns autores defendem que essa técnica pode ser utilizada no pós-operatório de gastrectomia, desde que haja avaliação criteriosa do paciente e cuidado com a técnica de VMNI a ser em- pregada. O uso de CPAP limitado a $7,5 \mathrm{cmH}_{2} \mathrm{O}$ ou mesmo PS + PEEP, desde que o valor de pico de pressão inspiratória não ultrapasse $10 \mathrm{cmH}_{2} \mathrm{O}$ e a PEEP não seja maior que $5 \mathrm{cmH}_{2} \mathrm{O}$, parece ser maneira segura de se empregar VMNI no período pós-operatório de cirurgia abdominal e ressecção pulmonar eletivas, na tentativa de evitarem-se complicações associadas à VMNI no pós-operatório, principalmente distensão gástrica e vômitos. O uso do capacete como interface parece ser uma opção à máscara facial para aqueles pacientes com necessidade de uso da sonda nasogástrica no período pós-operatório imediato.

Acredita-se, entretanto, que VMNI não deve ser utilizada no pós-operatório imediato de esofagectomia ou naqueles pacientes com distensão abdominal, náuseas e vômitos, deiscências, perfurações ou outras complicações operatórias do trato gastrintestinal.

\section{VMNI NA INSUFICIÊNCIA RESPIRATÓRIA PÓS-EX- TUBAÇÃO}

Recomendação: A VMNI não deve ser utilizada como método de resgate na insuficiência respiratória desenvolvida após a extubação, pois ela pode retardar a reintubação.

\section{Grau de Recomendação: A}

Comentário: Insuficiência respiratória após a extubação pode ocorrer mesmo após uma adequada condução do desmame e até o momento não há nenhum parâmetro objetivo que possa identificar os pacientes em risco. Cerca de $13 \%$ a $19 \%$ dos pacientes extubados necessitam de reintubação. Eles apresentam mortalidade sete vezes maior que os extubados com sucesso. Dentre as principais causas de reintubação estão a obstrução de vias aéreas superiores (edema de glote), fadiga muscular respiratória, edema cardiogênico, infecções, inabilidade de eliminar secreções, hipoxemia grave, alteração no estado mental e balanço hídrico positivo. Algumas séries de casos e estudos não aleatórios ${ }^{82-86}$ foram publicados, desde a década de 1990 , utilizando a VMNI para evitar a reintubação na falência respiratória pós-extubação, com resultados promissores. No entanto, dois estudos aleatórios ${ }^{87,88}$ não confirmaram o benefício da VMNI como método de resgate da insuficiência respiratória após extubação. Avaliando os dois estudos, foi alocado um total de 302 pacientes não selecionados com falência respiratória identificada dentro de 48 horas pós-extubação. Os pacientes foram aleatorizados em dois grupos; um grupo recebeu tratamento convencional (oxigênio, fisioterapia e broncodilatadores) e o outro recebeu VMNI por máscara facial 
associada ao tratamento convencional. Em ambos os estudos, não foram observadas, entre os dois grupos, diferenças significativas nas taxas de reintubação, no tempo de permanência e na mortalidade, tanto hospitalar quanto em UTI. Análise posterior, avaliando apenas os pacientes com hipercapnia (incluindo os portadores de DPOC), também não observou quaisquer diferenças. Baseando-se nesses dois estudos, pode-se concluir que a VMNI não foi eficaz em evitar a reintubação na falência respiratória que ocorreu após a extubação em grupos de pacientes não selecionados.

Recomendação: VMNI na falência respiratória pósextubação pode ter riscos e aumentar a mortalidade, principalmente se houver uma demora em se proceder à reintubação.

\section{Grau de Recomendação: A}

Comentário: Estudo multicêntrico aleatório, envolvendo 221 pacientes não selecionados com falência respiratória pós-extubação, observou maior mortalidade na UTI no grupo que recebeu VMNI em relação ao grupo que recebeu tratamento convencional $(25 \%$ versus $14 \%, p=0,048)^{88}$. Um dado extremamente importante observado nesse estudo foi que o intervalo de tempo entre o desenvolvimento da falência respiratória e a reintubação foi significativamente maior no grupo que recebeu VMNI em relação ao grupo que recebeu tratamento convencional ( 12 horas versus $2 \mathrm{~h} 30 \mathrm{~min}, \mathrm{p}=$ 0,02 ). Esse retardo na reintubação pode ter aumentado a mortalidade no grupo que recebeu VMNI. A causa do aumento da mortalidade nesses casos se deveu a vários mecanismos tais como isquemia cardíaca, aumento da fadiga muscular respiratória, pneumonite por aspiração e complicações da intubação de emergência.

Área de Controvérsia: Três estudos aleatórios avaliaram o uso precoce e sistemático da VMNI após a extubação como forma de prevenir a insuficiência respiratória pós-extubação, com resultados controversos. Jiang e col. ${ }^{89}$, avaliou 93 pacientes que foram extubados e imediatamente aleatorizados para receber VMNI com PS + PEEP por máscara facial ou oxigenoterapia. Não foram observadas diferenças significativas na taxa de reintubação entre os grupos. Esse estudo apresentou problemas na aleatorização, pois a maioria dos pacientes com extubação não programada recebeu VMNI, e esse foi o grupo que mais necessitou de reintubação. Nava e col. avaliaram 97 pacientes em ventilação mecânica por mais de 48 horas, considerados de alto risco para insuficiência respiratória pós-extubação (hipercapnia, insuficiência cardíaca congestiva, tosse ineficaz, secreção traqueobrônquica excessiva, mais de uma falência em tentativas de desmame, mais de uma comorbidade e obstrução de via aérea superior). Esses pacientes foram aleatorizados para receber VMNI ou tratamento convencional após a extubação. Aqueles que receberam VMNI tiveram menor taxa de reintubação (8,3\% versus $24,5 \%)$ e menor mortalidade na UTI90. No estudo de Ferrer, 162 pacientes que toleraram o teste de ventilação espontânea, mas considerados de alto risco para reintubação (idade $>65$ anos, falência cardíaca como causa da intubação ou APACHE II > 12 no dia da extubação), foram aleatorizados para 24 horas de VMNI com PS + PEEP ou oxigenoterapia, logo após a extubação. O desfecho primário foi redução da ocorrência de insuficiência respiratória pós-extubação. Esse desfecho foi menos freqüente no grupo que recebeu VMNI (16\% versus 33\% no grupo oxigenoterapia). A mortalidade na UTI também foi menor nesse grupo, porém não houve diferença da mortalidade em 90 dias. Uma comparação entre pacientes com e sem hipercapnia durante o teste de ventilação espontânea sugere benefício maior da VMNI nos pacientes hipercápnicos ${ }^{91}$.

\section{VMNI COMO ESTRATÉGIA DE DESMAME}

Recomendação: A VMNI através de máscara facial como estratégia de desmame pode ser utilizada em paciente com repetidas falhas no teste de respiração espontânea, porém as evidências de seu benefício ainda são consideradas insuficientes.

\section{Grau de Recomendação: B}

Comentário: Três grandes estudos aleatórios abordaram o uso da VMNI como técnica de desmame ${ }^{92-94}$. Nesses estudos, foram incluídos pacientes submetidos à ventilação mecânica por 24 a 48 horas e que, após atingirem os critérios para desmame, falharam no teste de ventilação espontânea. Esses pacientes foram aleatorizados em duas estratégias de desmame: um grupo foi extubado, e imediatamente submetido à VMNI por máscara facial e o outro grupo permaneceu intubado e submetido à ventilação com pressão de suporte em redução progressiva, ou à ventilação assistida-controlada associada a testes de ventilação espontânea através de tubo-T diariamente. Analisando os três estudos em conjunto, a duração média da VMNI foi de 13 a 24 horas/dia, nas 24 a 48 horas iniciais, aumentando-se, progressivamente, os intervalos sem suporte ventilatório. A VMNI foi aplicada através de máscara facial, com PS variando de 5 a $15 \mathrm{cmH}_{2} \mathrm{O}$ e PEEP de 4 a $5 \mathrm{cmH}_{2} \mathrm{O}$, objetivando $\mathrm{FR}<25$ irpm, troca gasosa adequada e conforto do paciente. Em dois desses estudos ${ }^{92,94}$, o grupo submetido à VMNI apresentou redução na du- 
ração da ventilação mecânica, na taxa de pneumonia e de choque séptico, proporcionando assim aumento na sobrevida em UTI e na sobrevida de 60 e 90 dias. A menor duração da ventilação mecânica observada no grupo que recebeu VMNI ainda permitiu redução na permanência em UTI e hospitalar. Um dos estudos $^{93}$ não demonstrou diferenças significativas entre os pacientes que receberam $\mathrm{VMNI}$ e o grupo controle, respectivamente, nas taxas de sucesso do desmame, complicações, estadia na UTI, estadia hospitalar e sobrevida em 3 meses.

Uma metanálise recente ${ }^{95}$ selecionou cinco ensaios controlados e aleatórios, considerados de moderada a boa qualidade, abordando o uso da VMNI como estratégia de desmame para pacientes intubados e submetidos à ventilação mecânica para tratamento de insuficiência respiratória. Foi incluído 171 pacientes, com predomínio de portadores de DPOC. A conclusão foi que a VMNI como estratégia de desmame reduziu a duração da intubação traqueal, a duração total do suporte ventilatório, a incidência de pneumonia associada ao ventilador ( $R R=0,28$, IC 95\% $=0,09-0,85)$, a permanência na UTI e hospitalar, e a mortalidade (RR $=0,41, \mathrm{IC} 95 \%=0,22-0,76)$. A análise do subgrupo de pacientes em ventilação mecânica por exacerbação de DPOC demonstrou que esses foram os que mais se beneficiaram do uso da VMNI como estratégia de desmame. Ainda assim, a evidência foi considerada insuficiente para recomendar seu uso rotineiro.

Dessa forma, a VMNI como estratégia de desmame foi considerada promissora, embora evidências do seu benefício clínico ainda sejam insuficientes.

\section{FLUXOGRAMA}

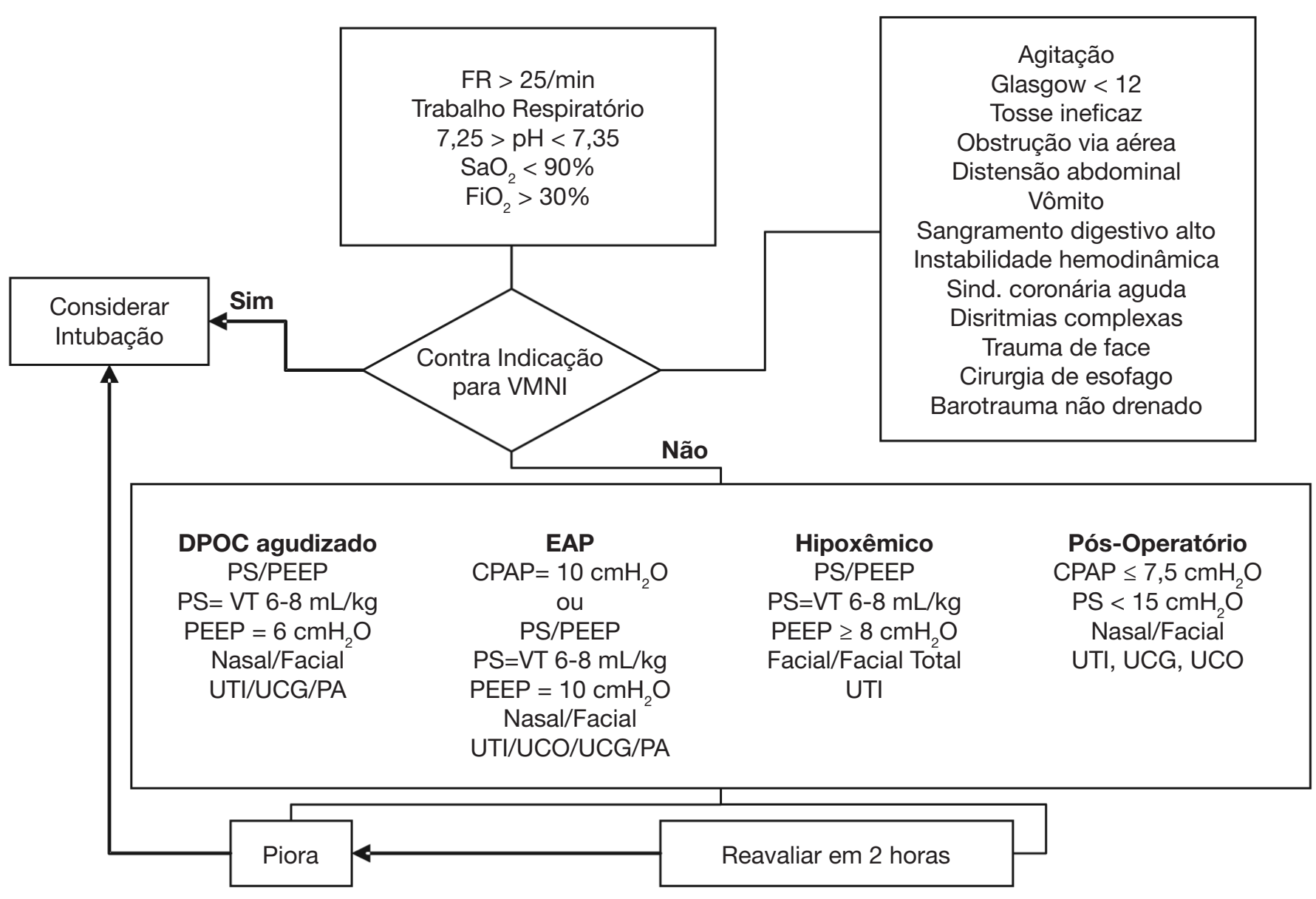




\section{REFERÊNCIAS}

01. International Consensus Conferences in Intensive Care Medicine: noninvasive positive pressure ventilation in acute Respiratory failure. Am J Respir Crit Care Med, 2001;163:283-291.

02. Wyatt J, Bellis F - British Thoracic Society guidelines on non-invasive ventilation. Emerg Med J, 2002;19:435.

03. Soo Hoo GW, Santiago S, Williams AJ - Nasal mechanical ventilation for hypercapnic respiratory failure in chronic obstructive pulmonary disease: determinants of success and failure. Crit Care Med, 1994;22:1253-1261.

04. Richards GN, Cistulli PA, Ungar RG et al - Mouth leak with nasal continuous positive airway pressure increases nasal airway resistance. Am J Respir Crit Care Med, 1996;154:182-186.

05. Martins De Araujo MT, Vieira SB et al - Heated humidification or face mask to prevent upper airway dryness during continuous positive airway pressure therapy. Chest, 2000;117:142-147.

06. Navalesi P, Fanfulla F, Frigerio P et al - Physiologic evaluation of noninvasive mechanical ventilation delivered with three types of masks in patients with chronic hypercapnic respiratory failure. Crit Care Med, 2000;28:1785-1790.

07. Kwok H, McCormack J, Cece R et al - Controlled trial of oronasal versus nasal mask ventilation in the treatment of acute respiratory failure. Crit Care Med, 2003;31:468-473.

08. Schettino GP, Chatmongkolchart S, Hess DR et al - Position of exhalation port and mask design affect $\mathrm{CO} 2$ rebreathing during noninvasive positive pressure ventilation. Crit Care Med, 2003;31:2178-2182.

09. Criner GJ, Travaline JM, Brennan KJ et al - Efficacy of a new full face mask for noninvasive positive pressure ventilation. Chest, 1994;106:1109-1115.

10. Antonelli M, Pennisi MA, Pelosi P et al - Noninvasive positive pressure ventilation using a helmet in patients with acute exacerbation of chronic obstructive pulmonary disease: a feasibility study. Anesthesiology, 2004;100:16-24.

11. Taccone $P$, Hess $D$, Caironi $P$ et al - Continuous positive airway pressure delivered with a "helmet": effects on carbon dioxide rebreathing. Crit Care Med, 2004;32:2090-2096.

12. Ferguson GT, Gilmartin M - CO2 rebreathing during BiPAP ventilatory assistance. Am J Respir Crit Care Med, 1995;151:1126-1135.

13. Lofaso F, Brochard L, Hang T et al - Home versus intensive care pressure support devices. Experimental and clinical comparison. Am J Respir Crit Care Med, 1996;153:1591-1599.

14. Bunburaphong $T$, Imanaka $H$, Nishimura $M$ et al - Performance characteristics of bilevel pressure ventilators: a lung model study. Chest, 1997;111:1050-1060.

15. Stell IM, Paul G, Lee KC et al - Noninvasive ventilator triggering in chronic obstructive pulmonary disease. A test lung comparison. Am J Respir Crit Care Med, 2001;164:2092-2097.

16. Vitacca M, Barbano L, D'Anna S et al - Comparison of five bilevel pressure ventilators in patients with chronic ventilatory failure: a physiologic study. Chest 2002;122(6):2105-14.

17. Hess DR. The evidence for noninvasive positive-pressure ventilation in the care of patients in acute respiratory failure: a systematic review of the literature. Respir Care, 2004;49:810-829.

18. Porta R, Appendini L, Vitacca M et al - Mask proportional assist vs pressure support ventilation in patients in clinically stable condition with chronic ventilatory failure. Chest, 2002;122:479-488.

19. Vitacca M, Clini E, Pagani M et al - Physiologic effects of early administered mask proportional assist ventilation in patients with chronic obstructive pulmonary disease and acute respiratory failure. Crit Care Med, 2000;28:1791-1797.

20. Wysocki M, Richard JC, Meshaka P - Noninvasive proportional assist ventilation compared with noninvasive pressure support ventilation in hypercapnic acute respiratory failure. Crit Care Med, 2002;30:323-329.

21. Masip J, Betbese AJ, Paez J et al - Non-invasive pressure support ventilation versus conventional oxygen therapy in acute cardiogenic pulmonary oedema: a randomised trial. Lancet, 2000;356(9248):2126-2132.

22. Park M, Sangean MC, Volpe Mde S et al - Randomized, prospective trial of oxygen, continuous positive airway pressure, and bilevel positive airway pressure by face mask in acute cardiogenic pulmonary edema. Crit Care Med, 2004;32:2407-2415.

23. Fu C, Caruso P, Lucatto JJ et al - Comparison of two flow generators with a noninvasive ventilator to deliver continuous positive airway pressure: a test lung study. Intensive Care Med, 2005;31:1587-1591.

24. Lightowler JV, Wedzicha JA, Elliott MW et al - Non-invasive positive pressure ventilation to treat respiratory failure resulting from exacerbations of chronic obstructive pulmonary disease: Cochrane systematic review and meta-analysis. BMJ, 2003;326(7382):185.

25. Keenan SP, Sinuff T, Cook DJ et al - Which patients with acute exacerbation of chronic obstructive pulmonary disease benefit from noninvasive positivepressure ventilation? A systematic review of the literature. Ann Intern Med, 2003;138:861-870.

26. Ram FS, Picot J, Lightowler $\mathrm{J}$ et al - Non-invasive positive pressure ventilation for treatment of respiratory failure due to exacerbations of chronic obstructive pulmonary disease. Cochrane Database Syst Rev, 2004(3): CD004104.

27. Bott J, Carroll MP, Conway JH et al - Randomised controlled trial of nasal ventilation in acute ventilatory failure due to chronic obstructive airways disease. Lancet, 1993;341(8860):1555-1557.

28. Brochard L, Mancebo J, Wysocki M et al - Noninvasive ventilation for acute exacerbations of chronic obstructive pulmonary disease. N Engl J Med, 1995;333:817-822.

29. Angus RM, Ahmed AA, Fenwick LJ et al - Comparison of the acute effects on gas exchange of nasal ventilation and doxapram in exacerbations of chronic obstructive pulmonary disease. Thorax, 1996;51:1048-1050.

30. Barbe F, Togores B, Rubi M et al - Noninvasive ventilatory support does not facilitate recovery from acute respiratory failure in chronic obstructive pulmonary disease. Eur Respir J, 1996;9:1240-1245.

31. Celikel T, Sungur M, Ceyhan B et al - Comparison of noninvasive positive pressure ventilation with standard medical therapy in hypercapnic acute respiratory failure. Chest, 1998;114:1636-1642.

32. Dikensoy O, Ikidag B, Filiz A et al - Comparison of non-invasive ventilation and standard medical therapy in acute hypercapnic respiratory failure: a randomised controlled study at a tertiary health centre in SE Turkey. Int J Clin Pract, 2002;56:85-88.

33. Plant PK, Owen JL, Elliott MW - Early use of non-invasive ventilation for acute exacerbations of chronic obstructive pulmonary disease on general respiratory wards: a multicentre randomised controlled trial. Lancet, 2000;355(9219):1931-1935.

34. Bardi G, Pierotello R, Desideri M et al - Nasal ventilation in COPD exacerbations: early and late results of a prospective, controlled study. Eur Respir J, 2000;15:98-104.

35. Collaborative Research Group of Noninvasive Mechanical Ventilation for Chronic Obstructive Pulmonary Disease. Early use of non-invasive positive pressure ventilation for acute exacerbations of chronic obstructive pulmonary disease: a multicentre randomized controlled trial. Chin Med J, 2005;118:2034-2040.

36. Meduri GU, Cook TR, Turner RE et al - Noninvasive positive pressure ventilation in status asthmaticus. Chest, 1996;110:767-774.

37. Soroksky A, Stav D, Shpirer I - A pilot prospective, randomized, placebocontrolled trial of bilevel positive airway pressure in acute asthmatic attack. Chest, 2003;123:1018-1025.

38. Ram FS, Wellington S, Rowe B et al - Non-invasive positive pressure ventilation for treatment of respiratory failure due to severe acute exacerbations of asthma. Cochrane Database Syst Rev, 2005(3):CD004360.

39. Rasanen J, Heikkila J, Downs $\mathrm{J}$ et al - Continuous positive airway pressure by face mask in acute cardiogenic pulmonary edema. Am J Cardiol, 1985;55:296-300.

40. Bersten AD, Holt AW, Vedig AE et al - Treatment of severe cardiogenic pulmonary edema with continuous positive airway pressure delivered by face mask. N Engl J Med, 1991;325:1825-1830.

41. Lin M, Yang YF, Chiang HT et al - Reappraisal of continuous positive airway pressure therapy in acute cardiogenic pulmonary edema. Short-term results and long-term follow-up. Chest, 1995;107:1379-1386.

42. Pang D, Keenan SP, Cook DJ et al - The effect of positive pressure airway support on mortality and the need for intubation in cardiogenic pulmonary edema: a systematic review. Chest, 1998;114:1185-1192.

43. L'Her E, Duquesne F, Girou E et al - Noninvasive continuous positive airway pressure in elderly cardiogenic pulmonary edema patients. Intensive Care Med, 2004;30:882-888

44. Masip J, Roque M, Sanchez B et al - Noninvasive ventilation in acute cardiogenic pulmonary edema: systematic review and meta-analysis. JAMA, 2005;294:3124-3130

45. Park M, Lorenzi-Filho G - Noninvasive mechanical ventilation in the treatment of acute cardiogenic pulmonary edema. Clinics, 2006;61:247-252.

46. Mehta S, Jay GD, Woolard RH et al - Randomized, prospective trial of bilevel versus continuous positive airway pressure in acute pulmonary edema. Crit Care Med, 1997;25:620-628.

47. Bellone A, Vettorello M, Monari A et al - Noninvasive pressure support ventilation vs. continuous positive airway pressure in acute hypercapnic pulmonary edema. Intensive Care Med, 2005;31:807-811. 
48. Nava S, Carbone G, DiBattista $\mathrm{N}$ et al - Noninvasive ventilation in cardiogenic pulmonary edema: a multicenter randomized trial. Am J Respir Crit Care Med, 2003;168:1432-1437.

49. Crane SD, Elliott MW, Gilligan P et al - Randomised controlled comparison of continuous positive airways pressure, bilevel non-invasive ventilation, and standard treatment in emergency department patients with acute cardiogenic pulmonary oedema. Emerg Med J, 2004;21:155-161.

50. Evans TW - International Consensus Conferences in Intensive Care Medicine: non-invasive positive pressure ventilation in acute respiratory failure. Organised jointly by the American Thoracic Society, the European Respiratory Society, the European Society of Intensive Care Medicine, and the Societe de Reanimation de Langue Francaise, and approved by the ATS Board of Directors, December 2000. Intensive Care Med, 2001;27:166-178.

51. Non-invasive ventilation in acute respiratory failure. Thorax, 2002:57:192-211.

52. Liesching T, Kwok H, Hill NS - Acute applications of noninvasive positive pressure ventilation. Chest, 2003;124:699-713.

53. Mehta S, Hill NS - Noninvasive ventilation. Am J Respir Crit Care Med, 2001;163:540-577.

54. Antonelli M, Conti G, Rocco M et al - A comparison of noninvasive positivepressure ventilation and conventional mechanical ventilation in patients with acute respiratory failure. N Engl J Med, 1998;339:429-435.

55. Ferrer M, Esquinas A, Leon M et al - Noninvasive ventilation in severe hypoxemic respiratory failure: a randomized clinical trial. Am J Respir Crit Care Med, 2003;168:1438-1444.

56. Keenan SP, Kernerman PD, Cook DJ et al - Effect of noninvasive positive pressure ventilation on mortality in patients admitted with acute respiratory failure: a meta-analysis. Crit Care Med, 1997;25:1685-1692.

57. Peter JV, Moran JL, Phillips-Hughes J et al - Noninvasive ventilation in acute respiratory failure--a meta-analysis update. Crit Care Med, 2002;30:555-562.

58. Keenan SP, Sinuff T, Cook DJ et al - Does noninvasive positive pressure ventilation improve outcome in acute hypoxemic respiratory failure? A systematic review. Crit Care Med, 2004;32:2516-2523.

59. Antonelli M, Conti G, Bufi M et al - Noninvasive ventilation for treatment of acute respiratory failure in patients undergoing solid organ transplantation: a randomized trial. JAMA, 2000;283:235-241.

60. Hilbert G, Gruson D, Vargas F et al - Noninvasive ventilation in immunosuppressed patients with pulmonary infiltrates, fever, and acute respiratory failure. N Engl J Med, 2001;344:481-487.

61. Confalonieri M, Calderini E, Terraciano S et al - Noninvasive ventilation for treating acute respiratory failure in AIDS patients with Pneumocystis carinii pneumonia. Intensive Care Med, 2002;28:1233-1238.

62. Confalonieri $\mathrm{M}$, Potena $\mathrm{A}$, Carbone $\mathrm{G}$ et al - Acute respiratory failure in patients with severe community-acquired pneumonia. A prospective randomized evaluation of noninvasive ventilation. Am J Respir Crit Care Med, 1999;160(5 Pt 1):1585-1591.

63. Auriant I, Jallot $A$, Herve $P$ et al - Noninvasive ventilation reduces mortality in acute respiratory failure following lung resection. Am J Respir Crit Care Med, 2001;164:1231-1235.

64. Rocker GM, Mackenzie MG, Williams B et al - Noninvasive positive pressure ventilation: successful outcome in patients with acute lung injury/ARDS. Chest, 1999;115:173-177.

65. Meduri GU, Fox RC, Abou-Shala $\mathrm{N}$ et al - Noninvasive mechanical ventilation via face mask in patients with acute respiratory failure who refused endotracheal intubation. Crit Care Med, 1994;22:1584-1590.

66. Benhamou D, Girault C, Faure C et al - Nasal mask ventilation in acute respiratory failure. Experience in elderly patients. Chest, 1992;102:912-917.

67. Chu CM, Chan VL, Wong IW et al - Noninvasive ventilation in patients with acute hypercapnic exacerbation of chronic obstructive pulmonary disease who refused endotracheal intubation. Crit Care Med, 2004;32:372-377.

68. Levy M, Tanios MA, Nelson D et al - Outcomes of patients with do-notintubate orders treated with noninvasive ventilation. Crit Care Med, 2004;32:2002-2007.

69. Schettino G, Altobelli N, Kacmarek RM - Noninvasive positive pressure ventilation reverses acute respiratory failure in select "do-not-intubate" patients. Crit Care Med, 2005;33:1976-1982.

70. Ricksten SE, Bengtsson A, Soderberg $\mathrm{C}$ et al - Effects of periodic positive airway pressure by mask on postoperative pulmonary function. Chest, 1986:89:774-781.

71. Celli BR, Rodriguez KS, Snider GL - A controlled trial of intermittent positive pressure breathing, incentive spirometry, and deep breathing exercises in preventing pulmonary complications after abdominal surgery. Am Rev Respir Dis, 1984;130:12-15.

72. Schuppisser JP, Brandli O, Meili U - Postoperative intermittent positive pressure breathing versus physiotherapy. Am J Surg, 1980;140:682-686.

73. Kindgen-Milles $D$, Buhl R, Gabriel A et al - Nasal continuous positive airway pressure: A method to avoid endotracheal reintubation in postoperative high-risk patients with severe nonhypercapnic oxygenation failure. Chest, 2000;117:1106-1111.

74. Varon J, Walsh GL, Fromm RE Jr - Feasibility of noninvasive mechanical ventilation in the treatment of acute respiratory failure in postoperative cancer patients. J Crit Care, 1998;13:55-57.

75. Ebeo CT, Benotti PN, Byrd RP Jr et al - The effect of bi-level positive airway pressure on postoperative pulmonary function following gastric surgery for obesity. Respir Med, 2002;96:672-676.

76. Joris JL, Sottiaux TM, Chiche JD et al - Effect of bi-level positive airway pressure (BiPAP) nasal ventilation on the postoperative pulmonary restrictive syndrome in obese patients undergoing gastroplasty. Chest, 1997;111:665-670.

77. Squadrone V, Coha M, Cerutti E et al - Continuous positive airway pressure for treatment of postoperative hypoxemia: a randomized controlled trial. JAMA, 2005;293:589-595.

78. Aguilo R, Togores $\mathrm{B}$, Pons $\mathrm{S}$ et al - Noninvasive ventilatory support after lung resectional surgery. Chest, 1997;112:117-121.

79. Gust R, Gottschalk A, Schmidt $\mathrm{H}$ et al - Effects of continuous (CPAP) and bi-level positive airway pressure (BiPAP) on extravascular lung water after extubation of the trachea in patients following coronary artery bypass grafting. Intensive Care Med, 1996;22:1345-1350.

80. Matte $\mathrm{P}$, Jacquet $\mathrm{L}$, Van Dyck $\mathrm{M}$ et al - Effects of conventional physiotherapy, continuous positive airway pressure and non-invasive ventilatory support with bilevel positive airway pressure after coronary artery bypass grafting. Acta Anaesthesiol Scand, 2000;44:75-81.

81. Pasquina P, Merlani P, Granier JM et al - Continuous positive airway pressure versus noninvasive pressure support ventilation to treat atelectasis after cardiac surgery. Anesth Analg, 2004;99:1001-1008.

82. Meduri GU, Conoscenti CC, Menashe P et al - Noninvasive face mask ventilation in patients with acute respiratory failure. Chest, 1989;95:865-870.

83. Hilbert G, Gruson D, Portel L et al - Noninvasive pressure support ventilation in COPD patients with postextubation hypercapnic respiratory insufficiency. Eur Respir J, 1998;11:1349-1353.

84. Munshi IA, DeHaven B, Kirton O et al - Reengineering respiratory support following extubation: avoidance of critical care unit costs. Chest, 1999;116:1025-1028

85. Kilger $\mathrm{E}$, Briegel $\mathrm{J}$, Haller $\mathrm{M}$ et al - Effects of noninvasive positive pressure ventilatory support in non-COPD patients with acute respiratory insufficiency after early extubation. Intensive Care Med, 1999;25:1374-1380.

86. Vitacca M, Ambrosino N, Clini E et al - Physiological response to pressure support ventilation delivered before and after extubation in patients not capable of totally spontaneous autonomous breathing. Am J Respir Crit Care Med, 2001;164:638-641.

87. Keenan SP, Powers C, McCormack DG et al - Noninvasive positive-pressure ventilation for postextubation respiratory distress: a randomized controlled trial. JAMA, 2002;287:3238-3244.

88. Esteban A, Frutos-Vivar F, Ferguson ND et al - Noninvasive positivepressure ventilation for respiratory failure after extubation. N Engl J Med, 2004;350:2452-2460.

89. Jiang JS, Kao SJ, Wang SN - Effect of early application of biphasic positive airway pressure on the outcome of extubation in ventilator weaning. Respirology, 1999;4:161-165.

90. Nava S, Gregoretti C, Fanfulla F et al - Noninvasive ventilation to prevent respiratory failure after extubation in high-risk patients. Crit Care Med, 2005:33:2465-2470.

91. Ferrer M, Valencia M, Nicolas JM et al - Early noninvasive ventilation averts extubation failure in patients at risk: a randomized trial. Am J Respir Crit Care Med 2006;173:164-70.

92. Nava S, Ambrosino N, Clini E et al - Noninvasive mechanical ventilation in the weaning of patients with respiratory failure due to chronic obstructive pulmonary disease. A randomized, controlled trial. Ann Intern Med, 1998; $128: 721-728$

93. Girault C, Daudenthun I, Chevron V et al - Noninvasive ventilation as a systematic extubation and weaning technique in acute-on-chronic respiratory failure: a prospective, randomized controlled study. Am J Respir Crit Care Med, 1999;160:86-92.

94. Ferrer M, Esquinas A, Arancibia $\mathrm{F}$ et al - Noninvasive ventilation during persistent weaning failure: a randomized controlled trial. Am J Respir Crit Care Med, 2003;168:70-76.

95. Burns KE, Adhikari NK, Meade MO - Noninvasive positive pressure ventilation as a weaning strategy for intubated adults with respiratory failure. Cochrane Database Syst Rev, 2003(4):CD004127. 